\section{Equilibrium exchange kinetics in n-alkyl-PEO polymeric micelles: single exponential relaxation and chain length dependence}

\author{
Thomas Zinn, ${ }^{a}$ Lutz Willner, ${ }^{* a}$ Reidar Lund, $\dagger^{b}$ Vitaliy Pipich ${ }^{c}$ and Dieter Richter ${ }^{a}$ \\ Received 22nd September 2011, Accepted 16th November 2011 \\ DOI: $10.1039 / \mathrm{c} 1 \mathrm{sm} 06809 \mathrm{a}$
}

\begin{abstract}
In this communication we present first results on the chain exchange kinetics of n-alkyl-PEO polymeric micelles by time-resolved small angle neutron scattering. We found that the rate strongly depends on the alkyl-chain length and that the relaxation function almost perfectly follows the single exponential decay predicted by theory. The key achievement of this study is the experimental verification that core block polydispersity accounts for the almost logarithmic time decay in block copolymer micelles as recently suggested by Choi et al. The results thus directly show that unimer exchange is the main mechanism for molecular exchange in block copolymer micelles.
\end{abstract}

An important issue in the understanding of the self-assembling behavior of diblock copolymers in selective solvents is the kinetics of chain exchange between individual micellar entities in thermodynamic equilibrium. ${ }^{1}$ The exchange can be monitored by timeresolved small angle neutron scattering (TR-SANS) applying a sophisticated contrast variation technique. Significant perturbations as often required for other methods here are limited to simple hydrogen/deuterium isotope labelling. ${ }^{2-9}$ In an earlier work by using this technique we have studied the chain exchange kinetics of poly (ethylene-alt-propylene)-poly(ethylene oxide) (PEP-PEO) micelles in water/N,N-dimethylformamide mixtures. ${ }^{4,5,10}$ There we found that the measured relaxation curves follow a logarithmic time dependence in contradiction to the single exponential decay predicted by the theory of Halperin and Alexander. ${ }^{11}$ A single exponential decay was also observed by dissipative particle dynamics simulation ${ }^{12}$ but, in addition to single unimer exchange, contributions from small aggregate fragmentation/merging and unequal size fusion/fission as additional kinetic mechanisms were found. In order to explain the

a Jülich Centre for Neutron Science JCNS (JCNS-1) \& Institute for Complex Systems (ICS-1), Forschungszentrum Jülich GmbH, 52425 Jülich, Germany. E-mail: l.willner@fz-juelich.de; Fax: +49 246161 2610; Tel: +492461618718

${ }^{b}$ Center of Material Physics (CFM)/Donostia International Physics Center (DIPC), University of the Basque Country, Paseo Manuel de Lardizabal 5, 20018 San Sebastian, Spain

'Jülich Centre for Neutron Science JCNS, Forschungszentrum Jülich GmbH, Outstation at FRM II, Lichtenbergstraße 1, 85747 Garching, Germany

$\dagger$ Current address: Department of Material Sciences \& Engineering. 225, Hearst Memorial Mining Building University of California, Berkeley, CA, $94720-1760$ logarithmic decay, Lund et al. ${ }^{4,5}$ discussed different mechanisms including possible effects of polydispersity but none of them finally could sufficiently explain the observed behavior. More recently Choi et $a l^{8}$ succeeded in describing the logarithmic relaxation by taking properly into account the polydispersity of the core forming block. Following these ideas also the data of Lund et al. ${ }^{7}$ could be successfully re-evaluated thereafter. As put forward by Choi et al. the enormous effect of polydispersity on the kinetics can be rationalized by a double exponential dependence of the exchange rate on the core chain length: $R(t)=\exp (-k t)$ with $k \sim \exp \left(-E_{d} / k_{B} T\right)$ where the activation energy $E_{a}$ is a function of the degree of polymerization $N$ of the core block. Thus polydispersity effects become crucial even though $M_{w} / M_{n}$ (where $M_{w}$ and $M_{n}$ the weight and number average molecular weight) is generally very small in micelle forming model polymers. However, direct experimental evidence is still missing due to the lack of suitable model compounds which should ideally consist of a monodisperse core polymer. As polymerization is a statistical process inherently leading to materials with a chain length distribution, monodisperse model compounds are difficult to realize. In this work we have studied poly(ethylene oxide) polymers (PEO) of about $5 \mathrm{~kg} \mathrm{~mol}^{-1}$ bearing long hydrophobic n-alkyl (octadecyl $\mathrm{C}_{18}$, tetracosyl $\mathrm{C}_{24}$ and triacontanyl $\mathrm{C}_{30}$ ) head groups as an approach to a monodisperse polymeric block: $M_{w} / M_{n}$ (n-alkyl) $=1$. Structurally, these so-called hydrophobically end-capped PEO derivatives can be considered as hybrids between block copolymers and $\mathrm{C}_{\mathrm{n}} \mathrm{E}_{\mathrm{m}}$ non-ionic surfactants. Numerous studies have been performed in recent years on these materials carrying either one $(\alpha)$ or two $(\alpha, \omega)$ aliphatic ${ }^{13}$ or fluorocarbon end-groups. ${ }^{14}$ In particular their association behavior leads to interesting rheological properties which are useful for technological applications. Most of the research was focussed on structural and rheological properties varying temperature, concentration $^{15}$ and alkyl chain length. ${ }^{16}$ A systematic study of the kinetics in particular equilibrium exchange kinetics have not yet been performed so far although a knowledge of the exchange rate is of importance to understand e.g. the formation of crystalline phases in starlike micellar systems or the viscoelastic properties of transient networks.

The polymers were prepared by living anionic polymerization of h-EO or d-EO in toluene at $95{ }^{\circ} \mathrm{C}$ for $24 \mathrm{~h}$ using $80: 20$ stoichiometric mixtures of 1-alkanols with their corresponding potassium-1-alkoxides, $\quad \mathrm{C}_{n} \mathrm{H}_{2 n+1}-\mathrm{OH} / \mathrm{C}_{\mathrm{n}} \mathrm{H}_{2 \mathrm{n}+1}-\mathrm{O}^{-} \mathrm{K}^{+}, \quad$ as initiating system. The polymers were characterised by size exclusion 
chromatography (SEC) in tetrahydrofuran/N,N-dimethylacetamide, $80: 15 \mathrm{v} / \mathrm{v}$, at $40{ }^{\circ} \mathrm{C}$ and ${ }^{1} \mathrm{H}-\mathrm{NMR}$ as described for PEP-PEO polymers in ref. 10 and 17 . The determined polymer characteristics are summarized in Table 1 . We explicitly emphasize that the given values for $M_{w} / M_{n}$ stem from the polydispersity of the PEO as the n-alkyl block is monodisperse.

The structural characterization was carried out by SANS at $\Phi=0.25 \%$ in full contrast, i.e. h- $\mathrm{C}_{\mathrm{n}}$-h-PEO in $\mathrm{D}_{2} \mathrm{O}$ and $\mathrm{h}-\mathrm{C}_{\mathrm{n}}-\mathrm{d}-$ PEO in $\mathrm{H}_{2} \mathrm{O}$. For the kinetic studies the concentration was $\Phi=$ $1 \%$ in a $\mathrm{H}_{2} \mathrm{O} / \mathrm{D}_{2} \mathrm{O}$ water mixture and for $\mathrm{C}_{24}$-PEO5 additionally $0.25 \%$ and $0.5 \%$ to delineate any concentration effects. The composition of the isotopic water mixture was taken to match the average scattering length density $\rho=N_{\text {avo }} \sum b_{i} /(d M)$ of the two differently labelled polymers. $N_{\text {avo }}$ denotes Avogadro's number, $\sum b_{i}$ the sum of the coherent scattering lengths of each individual atom of a solvent molecule or polymer repeat unit of molar mass, $M$, and $d$ the corresponding mass densities. For the final reference state of the kinetics premixed blends with $\Phi_{h h}=\Phi_{h d l d d}=0.5$ were prepared and dissolved in the corresponding $\mathrm{D}_{2} \mathrm{O} / \mathrm{H}_{2} \mathrm{O}$ water mixtures. The SANS experiments were carried out at KWS-1 and KWS-2 instruments at FRM-II Garching, Germany. For the structural characterization two instrumental set-ups were adopted covering a Q-range of $0.006 \AA^{-1} \leq Q \leq 0.2 \AA^{-1}$. Data reduction was done according to known procedures using qtiKWS software provided by JCNS to obtain the absolute normalized macroscopic differential scattering cross-section $\mathrm{d} \Sigma / \mathrm{d} \Omega$ in units of $\mathrm{cm}^{-1}$. The kinetic measurements were carried out at instrumental setting with a Q-range of $0.006 \AA^{-1} \leq Q \leq 0.05 \AA^{-1}$. A stopped-flow apparatus (BioLogic ${ }^{\mathrm{TM}}$ SFM-300) was employed for rapid and reproducible mixing of the two differently labelled micellar solutions. The stopped-flow apparatus is fully synchronized with data acquisition allowing for a time resolution of $50 \mathrm{~ms}$. The samples were typically mixed and measured 20 times. The data were combined to get an improved scattering statistics. In order to quantitatively analyse the micellar scattering, the data were evaluated by a sophisticated core-shell form factor. We consider alkyl chains forming a homogeneous spherical core of radius $R_{c}$ which is surrounded by a diffuse PEO corona. Neglecting structure factor contributions, the total macroscopic differential scattering cross section is given by

$$
\frac{\mathrm{d} \Sigma}{\mathrm{d} \Omega}(Q)=\frac{\phi}{N_{a g g} V_{C n-P E O}} P(Q)+\frac{\mathrm{d} \Sigma}{\mathrm{d} \Omega}_{b l o b}(Q)
$$

where $N_{\text {agg }}$ denotes the aggregation number of the micelle and $V_{C n-P E O}$ is the molar volume of the polymer. $\mathrm{d} \Sigma / \mathrm{d} \Omega_{\text {blob }}(Q)$ accounts for the so called "blob scattering" as defined in a previous publication, ${ }^{17}$ in order to satisfactorily fit the high $Q$ values.

Table 1 Polymer and equilibrium micellar characteristics

\begin{tabular}{llllllll}
\hline Polymer & type & $M_{n / \mathrm{PEO}} / \mathrm{g} \mathrm{mol}^{-1}$ & $N_{\mathrm{PEO}}$ & $M_{w} / M_{n}$ & $N_{\mathrm{agg}}$ & $R_{m} / \AA$ & $R_{c} / \AA$ \\
\hline $\mathrm{C}_{18}-\mathrm{PEO}$ & hh & 4000 & 91 & 1.05 & 27 & 81 & 15 \\
& dd & 4020 & 84 & 1.04 & 27 & 79 & 15 \\
$\mathrm{C}_{24}-\mathrm{PEO}$ & hh & 4180 & 95 & 1.03 & 71 & 103 & 23 \\
& hd & 4240 & 88 & 1.05 & 79 & 100 & 24 \\
$\mathrm{C}_{30}-\mathrm{PEO}$ & hh & 4300 & 98 & 1.03 & 122 & 113 & 29 \\
& hd & 4280 & 89 & 1.03 & 111 & 107 & 28 \\
\hline
\end{tabular}

Following Pedersen and Svaneborg, ${ }^{18}$ the micellar form factor, $P(Q)$, for a spherical geometry can be written as

$$
\begin{aligned}
& P(Q)=\left(\rho_{C n}-\rho_{0}\right)^{2} N_{\text {agg }}^{2} V_{C n}^{2} A_{c}^{2}(Q) \\
& +\left(\rho_{P E O}-\rho_{0}\right)^{2} N_{\text {agg }}\left(N_{\text {agg }}-\frac{1}{1+v}\right) V_{P E O}^{2} A_{s h}^{2}(Q) \\
& +2\left(\rho_{C n}-\rho_{0}\right)\left(\rho_{P E O}-\rho_{0}\right) N_{\text {agg }}^{2} V_{C n} V_{P E O} A_{c}(Q) A_{s h}(Q)
\end{aligned}
$$

where $\rho_{c / s h}$ is the scattering length density and $V_{C n / P E O}$ is the volume either of the alkyl chain, core (c), or the PEO corona (sh). In eqn (2) $A_{c}(Q)$ and $A_{s h}(Q)$ are the scattering amplitude of core and shell (corona), respectively. While $A_{c}(Q)$ is modelled by a homogeneously filled sphere of constant density, the scattering amplitude of the corona $A_{s h}(Q)$ comprises a starlike density profile: $n_{s h}(r)=r^{-4 / 3} /(1+$ $\left.\exp \left(\left(r-R_{m}\right) /\left(\sigma_{m} R_{m}\right)\right)\right) ; R_{m}$ denotes the micellar radius and $\sigma_{m}$ the smearing of the corona surface. Further details of this model are described in previous publications. ${ }^{10,17}$

For evaluation of the kinetic data, the time dependent scattering pattern obtained after mixing hd and hh type micelles were modelled by modifying the above core-shell model with a time dependent contrast as outlined in ref. 10. Different from earlier studies where hh and dd micelles were mixed, in the present case the only time dependent factor originates from a change of the contrast for the PEO corona, as the core always consists of h-alkyls, i.e. we have to replace $\rho_{P E O} \rightarrow$ $\rho_{P E O}(t)$ in eqn (2). Hence for the PEO in the corona, the exchange of chains will change the mean scattering length density according to:

$$
\rho_{\mathrm{PEO}}^{i}(t)= \begin{cases}\rho_{\mathrm{h}-\mathrm{PEO}} f(t)+\rho_{\mathrm{d}-\mathrm{PEO}}(1-f(t)) & \mathrm{h}-\text { type } \\ \rho_{\mathrm{h}-\mathrm{PEO}}(1-f(t))+\rho_{\mathrm{d}-\mathrm{PEO}} f(t) & \mathrm{d}-\text { type }\end{cases}
$$

where the excess function $f(t)$ is restricted to the range: $0.5 \leq f(t) \leq 1$. The complete time dependent scattered intensity can then be calculated by inserting eqn (3) for the hh and hd type micelles into eqn (2) and taking the average:

$$
\frac{\mathrm{d} \Sigma}{\mathrm{d} \Omega}(Q, t)=\frac{1}{2}\left(\frac{\mathrm{d} \Sigma^{\mathrm{hh}}}{\mathrm{d} \Omega}(Q, t)+\frac{\mathrm{d} \Sigma^{\mathrm{hd}}}{\mathrm{d} \Omega}(Q, t)\right)
$$

Structural data of the n-alkyl-PEO micelles are shown Fig. 1. The data are normalized by concentration and contrast according to, $C=\Delta \rho^{2} / \varphi N_{a v o}$. While for the $\mathrm{C}_{18}$-PEO5 the scattering profiles of hh and dd micelles are identical over the entire Q-range differences are observed at intermediate $\mathrm{Q}$ for $\mathrm{C}_{24}-\mathrm{PEO} 5$ and $\mathrm{C}_{30}-\mathrm{PEO} 5$ micelles. These differences arise from the fact that the core is proteated for both types of micelles. In the case of the fully proteated material in $\mathrm{D}_{2} \mathrm{O}$ both core and corona contribute to the scattering intensity, while for the deuterated material in $\mathrm{H}_{2} \mathrm{O}$ only the shell is visible. The scattering data were fitted with the core-shell model as defined above. Corresponding fits are shown as solid lines in Fig. 1. The resulting micellar parameters are summarized in Table 1. The determined values reveal that the micellar structure is almost independent of labelling. It should be noted at this point that similar micellar properties are an important prerequisite for the kinetic study. It is further observed that $N_{a g g}, R_{c}$ and $R_{m}$ systematically increase with the no. of carbons in the alkyl chain. This feature, already known from non-ionic surfactants, may be rationalized by packing arguments. The found aggregation behavior is in agreement with results obtained by Sommer and Pedersen commercial material (Brij700). ${ }^{19}$ The structure, however, will not be further discussed here as we will focus on the kinetics in this 


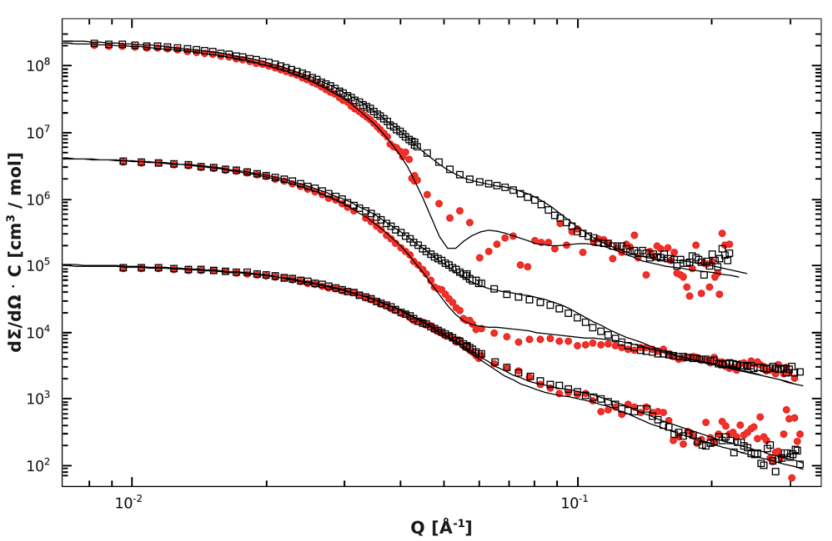

Fig. 1 Normalized scattering curves of deuterated (bullets) and proteated (open squares) in $\mathrm{H}_{2} \mathrm{O}$ or $\mathrm{D}_{2} \mathrm{O}$ at $\varphi=0.25 \%$. From top to bottom: $\mathrm{C}_{30}-\mathrm{PEO} 5, \mathrm{C}_{24}-\mathrm{PEO} 5$ and $\mathrm{C}_{18}-\mathrm{PEO} 5$ micelles. $\mathrm{C}_{24}$ and $\mathrm{C}_{30}$ data are off-set by arbitrary factors.

communication. The kinetics of the three different n-alkyl-PEO polymeric micelles were investigated by TR-SANS experiments. The accumulated detector count rates of several single shots as a function of time after mixing are shown in Fig. 2. This representation clearly demonstrates the enormous influence of the nalkyl chain length on the exchange kinetics in aqueous solution: Micelles with the longest n-alkyl block, $\mathrm{C}_{30}$, do not show any exchange over almost five orders in time up to $1000 \mathrm{~s}$. In the case of $\mathrm{C}_{18}$-PEO5 the intensity of the first measurement is already identical to the intensity of the final state as illustrated by the solid line. Hence, we can conclude that the time to accomplish randomization of $\mathrm{d}$ and $\mathrm{h}$ chains in each micelle is faster than the dead time of the stopped-flow instrument and therefore practically unresolvable at this temperature. A continuous decay of the count rate within the experimental time window of $100 \mathrm{~s}$ is observed only for the $\mathrm{C}_{24^{-}}$ PEO5. Due to the exchange of $\mathrm{h}$ - and d-chains the contrast becomes gradually smaller and consequently one observes a decrease of the macroscopic differential scattering cross section $\mathrm{d} \Sigma / \mathrm{d} \Omega(Q)$ as depicted for selected times in Fig. 3. We see that

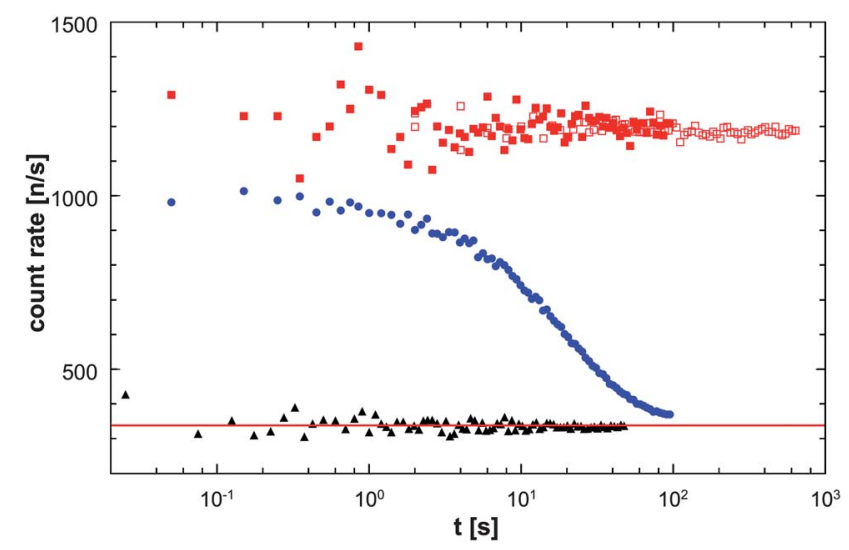

Fig. 2 Detector count rates as a function of time after mixing: $\mathrm{C}_{18^{-}}$ PEO5 (triangles), $50 \mathrm{~ms} ; \mathrm{C}_{24}-\mathrm{PEO} 5$ (circles) $100 \mathrm{~ms}$; $\mathrm{C}_{30}-\mathrm{PEO} 5$ (boxes): filled $100 \mathrm{~ms}$ and open $1000 \mathrm{~ms}$ time resolution. The line illustrates the final state of $\mathrm{C}_{18}-\mathrm{PEO}$. $\mathrm{d} \Sigma / \mathrm{d} \Omega(Q)$ reaches the intensity of the premixed randomized blend $(t=\infty)$ after approximately $95 \mathrm{~s}$. The evolution of scattering curves displays a non-trivial behavior with a minimum developing at higher $\mathrm{Q}$ values. This minimum is the result of the interference term between the protaeted core and corona changing its scattering length density in time. The scattering model describes this feature rather accurately. The TR-SANS data were also quantitatively evaluated by determining the relaxation function $R(t)$ according to

$$
R(t)=\sqrt{\frac{I(t)-I_{\infty}}{I(0)-I_{\infty}}}
$$

where $I(\mathrm{t})=\int I(Q, t) d Q$ is the integral intensity at a given time, $I_{\infty}$ is the intensity of the premixed blend representing the final state. $I(0)$ is the initial intensity of the first measurement after mixing where not yet a significant exchange of chains has taken place. Alternatively, we have evaluated the TR-SANS data using the time dependent core-shell model in order to account for the differences in the scattering curves at intermediate $Q$. As mentioned before, the evaluation relies on the decrease of contrast of the PEO corona $\Delta \rho_{\mathrm{PEO}}(t)$ by chain exchange. The scattered intensities $\mathrm{d} \Sigma / \mathrm{d} \Omega(Q, t)$ were fitted with the whole scattering profile using the fraction of either deuterated or proteated exchanged chains $f(t)$ as fit parameter. All other parameters are known from the structural evaluation of the scattering curves under full contrast. The results of the fits are shown as solid lines in Fig. 3. In analogy to the relaxation function $R(t), f_{\operatorname{exch}}(t)$ is defined as

$$
f_{\text {exch }}(t)=\frac{f(t)-f_{\infty}}{f(0)-f_{\infty}}
$$

The two relaxation functions $R(t)$ and $f_{\text {exch }}(t)$ for $\mathrm{C}_{24}-\mathrm{PEO} 5$ are plotted in a semi-log representation in Fig. 4. In this representation both methods show a linear dependence revealing the predicted single exponential decay: $R(t)=\exp (-t / \tau)$. This result clearly differs from previously found kinetics on PEP1-PEO1 ${ }^{10}$ and PEP1PEO20 $0^{4,5}$ block copolymer micelles which show a logarithmic time dependence over several orders of magnitude in time. The same observation was later made by Choi et al. ${ }^{8}$ on PS-PEP block copolymer micelles in squalane. Furthermore, they observed an

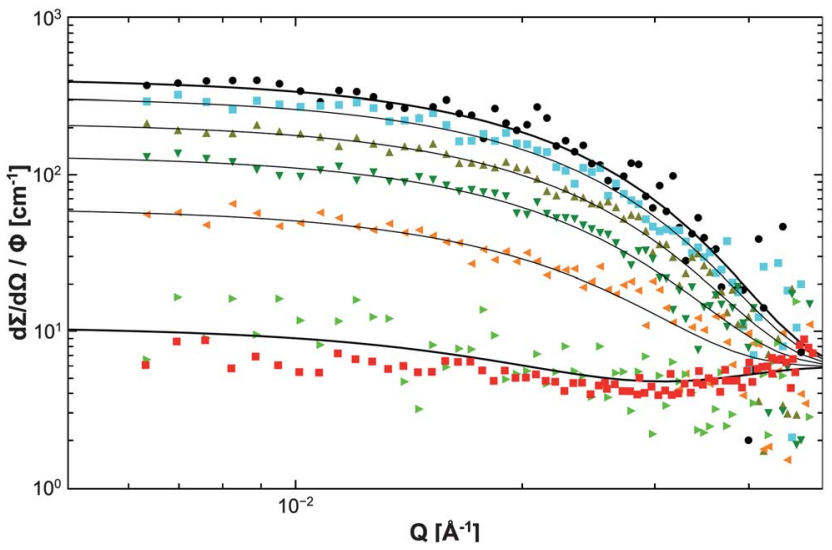

Fig. 3 Time evolution of normalized absolute scattering cross section of $\mathrm{C}_{24}$-PEO5 micelles after mixing. Solid lines display fits of the core-shell model with $f(t)$ as variable parameter. Time evolution from top to bottom: $\mathrm{t}=0.05 \mathrm{~s}, 5.6 \mathrm{~s}, 14.7 \mathrm{~s}, 25.2 \mathrm{~s}, 42.4 \mathrm{~s}, 95.0 \mathrm{~s}$. 
extensive effect of the core block molecular weight on the relaxation rates leading to the conclusion that modest polydispersity causes the approximate logarithmic time decay. In fact, by considering the actual polydispersity the logarithmic relaxation could be satisfactorily described. ${ }^{8}$ The single exponential decay as well as the strong dependence on the alkyl chain length of the monodisperse model systems found in this work impressively confirm this assumption. The extracted relaxation time, $\tau=44 \mathrm{~s}$, is almost independent of the evaluation method, $R(t)$ and $f_{\text {exch }}(t)$. Apparently, possible deviations arising from differences in the core-shell scattering profiles of hh and hd micelles are not yet pronounced as the kinetic runs were performed at rather low Q. The inset of Fig. 4 shows the concentration dependent measurements at $\varphi=0.25,0.5$ and $1 \%$ polymer volume fraction. Independent of $\varphi$ all relaxation curves fall on top of each other as it was already found for PEP-PEO micelles in water/DMF mixtures. ${ }^{5}$ In this context we confirm our previous result that single chain exchange is the dominant mechanism in the dilute concentration limit. This is in agreement with the Aniansson and Wall mechanism describing the equilibrium kinetics in lowmolecular weight surfactant micelles solely by expulsion/insertion of unimers. ${ }^{20}$ Concentration independent kinetics in dilute solutions were also found lately by dissipative particle dynamics simulation studies. ${ }^{12}$ Significant contributions from parallel existing mechanisms, e.g. fission and fusion are only observed at noticeably higher concentrations. Finally, we can compare the characteristic time $\tau$ with a calculated value obtained from a semi-empirical relation of Berret et al. $.^{14} \tau=3.3 \times 10^{-9} \exp (0.88 n)$ s. The characteristic times of this relation stem from linear rheology of transient networks formed by $(\alpha, \omega)$ hydrophobically end-capped PEOs. For $n=24$ a value of $5 \mathrm{~s}$ is calculated which is, considering the two completely different approaches, in fair agreement with our result. From the Berret equation also the characteristic times of $\mathrm{C}_{18}$-PEO5 and $\mathrm{C}_{30^{-}}$ PEO5 can be calculated which are $\tau=0.025 \mathrm{~s}$ and $\tau=1000 \mathrm{~s}$, respectively. Accordingly, a visible decay within the experimental time window would have been expected. Apparently, the effect of n-alkyl length on the relaxation behavior is stronger than expected from rheology. The rheological measurements, however, are usually performed at higher concentrations where the transient networks

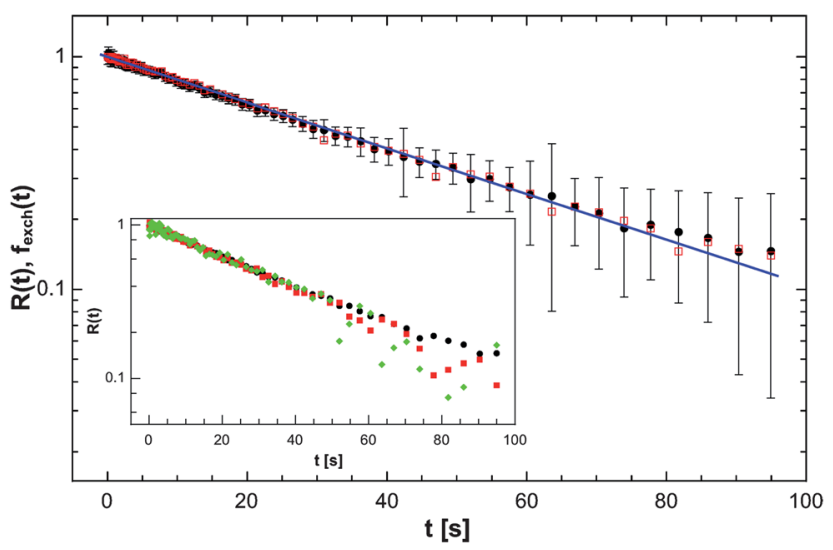

Fig. 4 Relaxation function $R(t)$ (bullets) and $f_{\text {exch }}(t)$ (open squares) of $\mathrm{C}_{24}$-PEO5 in a semi-log plot at room temperature. The line depicts the single exponential fit curve $R(t)$ for $\varphi=1 \%$. Inset layer showing relaxation curves for $0.25 \%$ (diamonds), $0.5 \%$ (squares) and $1 \%$ (circles) polymer volume fraction. are formed. A significant slowing down of the kinetics at higher concentrations compared to dilute solutions were observed recently by Choi et al. ${ }^{9}$ and subsequently explained by Halperin. ${ }^{21}$ Such an effect would lead to a larger discrepancy but still needs to be evaluated for the current monodisperse system. Also different temperatures may lead to significant deviations.

In any case we should underline that the TR-SANS technique yields direct access to the pure equilibrium kinetics. A correlation of the characteristic times obtained by rheology and TR-SANS would be useful to predict macroscopic properties of a transient network. In conclusion, we have studied the molecular exchange of n-alkyl hydrophobically end-capped PEOs in water by TR-SANS. This system can be regarded as a monodisperse block copolymer system or a hybrid between surfactants and polymers. Long n-alkyl groups of 18,24 , and 30 carbons were taken as monodisperse substitutes of a hydrophobic polydisperse polymer block. Our data confirm the single exponential time decay which is in coincidence with the prediction of the Aniansson and Wall model for fast unimer exchange between the micelles limited by unimer expulsion. The findings of this study support the assumption that polydispersity lead to the logarithmic relaxation in block copolymer micelles. Further systematic studies are planned for future work varying temperature, concentration and n-alkyl/PEO chain length in this model system.

\section{References}

1 T. Nicolai, O. Colombani and C. Chassenieux, Soft Matter, 2010, 6, 3111-3118.

2 L. Willner, A. Poppe, J. Allgaier, M. Monkenbusch and D. Richter, Europhys. Lett., 2001, 55, 667.

3 Y.-Y. Won, H. T. Davis and F. S. Bates, Macromolecules, 2003, 36, 953-955.

4 R. Lund, L. Willner, J. Stellbrink, P. Lindner and D. Richter, Phys. Rev. Lett., 2006, 96, 068302.

5 R. Lund, L. Willner, D. Richter and E. E. Dormidontova, Macromolecules, 2006, 39, 4566-4575.

6 R. Lund, L. Willner, D. Richter, H. Iatrou, N. Hadjichristidis and P. Lindner, Journal of Applied Crystallography, 2007, 40, 327-331.

7 R. Lund, L. Willner, J. Stellbrink, P. Lindner and D. Richter, Phys. Rev. Lett., 2010, 104, 049902.

8 S.-H. Choi, T. P. Lodge and F. S. Bates, Phys. Rev. Lett., 2010, 104, 047802.

9 S.-H. Choi, F. S. Bates and T. P. Lodge, Macromolecules, 2011, 44, 3594-3604.

10 R. Lund, L. Willner, V. Pipich, I. Grillo, P. Lindner, J. Colmenero and D. Richter, Macromolecules, 2011, 44, 6145-6154.

11 A. Halperin and S. Alexander, Macromolecules, 1989, 22, 2403-2412.

12 Z. Li and E. E. Dormidontova, Soft Matter, 2011, 7, 4179-4188.

13 E. Beaudoin, O. Borisov, A. Lapp, L. Billon, R. C. Hiorns and J. Franois, Macromolecules, 2002, 35, 7436-7447.

14 J.-F. Berret, D. Calvet, A. Collet and M. Viguier, Curr. Opin. Colloid Interface Sci., 2003, 8, 296-306.

15 F. Renou, L. Benyahia, T. Nicolai and O. Glatter, Macromolecules, 2008, 41, 6523-6530.

16 F. Renou, T. Nicolai, E. Nicol and L. Benyahia, Langmuir, 2009, 25, 515-521.

17 R. Lund, V. Pipich, L. Willner, A. Radulescu, J. Colmenero and D. Richter, Soft Matter, 2011, 7, 1491-1500.

18 J. S. Pedersen and C. Svaneborg, Curr. Opin. Colloid Interface Sci., 2002, 7, 158-166.

19 C. Sommer, J. S. Pedersen and V. M. Garamus, Langmuir, 2005, 21, $2137-2149$.

20 E. a. G. Aniansson, S. N. Wall, M. Almgren, H. Hoffmann, I. Kielmann, W. Ulbricht, R. Zana, J. Lang and C. Tondre, J. Phys. Chem., 1976, 80, 905-922.

21 A. Halperin, Macromolecules, 2011, 44, 5072-5074. 